of these congenital EOM fibrosis syndromes is more diffuse than previously recognized and extends beyond the extraocular muscles and involves also the cranial nerves and brain stem.

\title{
CSF CIRCULATION AND CONGENITAL HYDROCEPHALUS
}

A new model of the cerebrospinal fluid (CSF) circulation, involving brain capillaries as the main site of CSF absorption, is proposed from the Karolinska MR Research Center and Department of Neuroradiology, Karolinska Hospital, Stockholm, Sweden. In communicating hydrocephalus, a decrease of CSF flow through the foramen magnum demonstrated by MRI is explained by restricted expansion of the intracranial arteries. In the new classification, communicating hydrocephalus is called "restricted arterial pulsation (RAP) hydrocephalus," a primary hemodynamic disturbance, caused by any process that decreases compliance of arteries, eg ectasia, arteritis, spasm. In obstructive hydrocephalus, ventricular dilatation causes compression of cortical veins, a secondary hemodynamic disturbance called "venous

congestion (VC) hydrocephalus." Pharmacological treatment for hydrocephalus should consist of selective venous constrictor drugs such as dihydroergotamine. (Greitz D, Greitz T, Hindmarsh T. A new view on the CSFcirculation with the potential for pharmacological treatment of childhood hydrocephalus. Acta Paediatr Feb 1997;86:125-132). (Respond: Dr D Greitz, Karolinska MR Research Center, Karolinska Hospital, S-171 76, Stockholm, Sweden).

COMMENT. The authors caution that treatment of hydrocephalus with dihydroergotamine should not be employed in children until more studies are completed in adults. The new hemodynamic mechanism for hydrocephalus is more in keeping with studies involving flow sensitive MR imaging and radionuclide cisternography. As noted by Whitelaw A, in an editorial commentary, one of the strongest arguments against the conventional model of CSF circulation and absorption is the absence of Pacchionian granulations in children less than 12 months of age. However, the new hemodynamic concept is only a hypothesis which needs to be proven by further studies.

\section{MOVEMENT DISORDERS}

\section{DYSTONIA AND WRITER'S CRAMP TRIGGERED BY EXERCISE}

An 11-year-old girl with a gait disturbance and foot dystonia, especially after exertion at the end of the day, and handwriting difficulty with cramps after a short school exercise, was evaluated at the Neuropaediatric Unit, CHUV, Lausanne, Switzerland. Her gait was better in the morning and after rest, but when lying down, her feet were inverted and plantar flexed in a dystonic posture. Treatment with L-dopa, $100 \mathrm{mg}$, and benzerazide $25 \mathrm{mg}, 2$ to 3 times a day, relieved all symptoms and signs, but dystonia returned when L-dopa was discontinued. The mother had parkinsonism relieved by L-dopa. (Deonna T, Roulet E, Ghika J, Zesiger P. Dopa-responsive childhood dystonia: a forme fruste with writer's cramp, triggered by exercise. Dev. Med Child Neurol Jan 1997;39:49-53). (Respond: Dr Thierry-W Deonna, Neuropaediatric Unit, CHUV, 1011 Lausanne, Switzerland).

COMMENT. Children presenting with dysgraphia, fatigue, and cramps should be examined for possible dopa responsive dystonia, especially if the symptoms are associated with a gait disturbance at the end of the school day. 